\title{
The Self-Growing Concept - A novel Framework developed by the FP7 CONSERN Project
}

\author{
N. Alonistioti ${ }^{1}$, E. Schulz ${ }^{2}$, G. Koudouridis ${ }^{3}$, S. Delaere ${ }^{4}$, J. Declerk ${ }^{5}$, M. Stamatelatos ${ }^{1}$, \\ M. Emmelmann ${ }^{6}$, Ioannis P. Chochliouros ${ }^{7}$, M. Mueck ${ }^{8}$ \\ ${ }^{1}$ National and Kapodistrian Univeristy of Athens, Greece, ${ }^{2}$ Huawei, Germany, ${ }^{3}$ Huawei, Sweden, ${ }^{4}$ IBBT, Belgium, ${ }^{5}$ IMEC, Belgium, \\ ${ }^{6}$ Fraunhofer FOKUS, Germany, ${ }^{7}$ Hellenic Telecommunications Organization S.A. (OTE), Greece, ${ }^{8}$ Infineon Technologies, Germany
}

\begin{abstract}
The CONSERN project (COoperative aNd Self growing Energy awaRe Networks) develops the novel SelfGrowing concept. It is part of the European Commission's FP7 research initiative focusing on the following two major research directions: i) Solutions for optimised energy and power consumption in a small scale, purpose-driven network through balancing autonomic and cooperative approaches and ii) mechanisms for the self-evolvement of the network towards a large-scale, multi-purpose network. This paper will detail mechanisms developed within CONSERN that allow an on-going integration of existing networks in an ever-growing, complex environment, applying continuous self-optimization mechanisms and guaranteeing a minimized level of power-consumption, resource usage efficiency.
\end{abstract}

Keywords- Cognitive Radio (CR), Energy Efficiency, SelfGrowing Mechanism

\section{INTRODUCTION}

The European Commission funded FP7 CONSERN project aims at developing a novel paradigm for dedicated, purposedriven small scale wireless networks characterized by a service-centric evolutionary approach introduced here as an energy-aware Self-Growing network: In future distributed systems, the emergence of mechanisms achieving robust, predictable and self-adaptive behaviour for large-scale networked systems will be an important evolution step. In this context, key challenges of CONSERN lie in the efficient cooperation of heterogeneous elements in order to provide advanced problem solving capabilities and improved services. Furthermore, innovations for low energy are considered a fundamental parameter in the efforts to combat climate change and to achieve sustainable economic growth. Low energy solutions create an attractive business case by offering significant benefits in terms of operational cost, long-term product reliability, sustainability, and increased lifetime of wireless or mobile elements. For this purpose, a promising path lies in the study and development of energy-aware distributed and cooperating systems for monitoring and control, in particular based on wireless sensor networks for providing radio and environmental context information.

However, energy efficient and dependable operation at the level of cooperating wireless elements, network compartments and networks as a whole is becoming an increasingly difficult objective, given the ever-increasing complexity in heterogeneous telecommunication environments. In this context, the evolution of mechanisms that will cope with energy-aware and dependable cooperation of wireless elements becomes a fundamental enabler for future heterogeneous distributed systems.

Current developments towards more energy efficient network components are often introduced in the context of general network evolutions implemented by operators, for example by introducing more flexible base stations, more advanced cooling systems at the sites of these base stations, and more energy efficient transmission techniques for various wireless and wired (e.g. xDSL optimisation, DSLAM improvements) technologies [1]. However, there is a need for introducing such mechanisms in less generic markets, and creating solutions for smaller scale or specialized targets not served by these 'one-size-fits-all', large scale improvements.

Concerning energy efficiency, several approaches in the energy consumption field have been investigated. In [2] the power consumption is optimised through the use of a low power secondary radio, to wake up the higher power radio targeting on-demand paging cases. System design using Petrinet formalisms has been proposed in [3]. Power consumption of such implementations has been a concern, but the methodology has chiefly been used as a simulation technique [4] that models software at the instruction level, optionally enhanced with experimentally captured data [5]. Other research [6] has begun to apply formal analysis properties of Petri-nets to power control. Higher level models such as live sequence charts [7] or Hume specifications [8] have also been used, with these approaches concentrating mainly on handling liveness, execution time, and heap bounding properties. Moreover, [9] takes a power consumption focused approach, using a formalism called action systems to estimate power usage in systems comprised of composed processes. However, most of the previous works either address specific applications or have limited degrees of freedom for power management since generic run-time self-adaptation is not considered.

This paper provides an overview of the CONSERN project by combining initial key technical results regarding a selfgrowing framework [10] as well as functional requirements and enablers for self-growing energy aware systems [11] as derived from selected use cases and scenarios [6]. Besides key technical research, business modelling and validation by a proof-of-concept platform are key elements of CONSERN. It will be shown how novel self-growing based concept will lead to a dramatically increased level of efficiency. 
The sequel of this paper is organized as follows. Section II gives further details on the self-growing concept. Section III further details key self-growing directions while Section IV indicates further CONSERN working plans. Section V finally gives a conclusion.

\section{THE SELF-GROWING CONCEPT}

A Self-Growing network (Fig. 1) is set up on-demand, dedicated to a single purpose (energy-efficient networking, spectrum efficient cooperations, monitoring and/or controlling with a focus on large scale) including for example construction sites, delivering wireless services within a complex home/office environment requiring network parameter negotiation with a multitude of neighbouring networks, etc. During its lifecycle, it can evolve to serve several different objectives as needed, such as providing general voice and data communications, integrating sensor networks in the vicinity or supporting safety of life applications under exceptional situations. In the course of this it may coexist and cooperate with other wireless networks of distinct owners and interest groups evolving in the deployment area towards using or augmenting existing capacity. The sensor networks may serve a multitude of purposes, including environmental sensing, radio parameter sensing, etc. Interoperability with existing network infrastructures and wireless standards enables geographical and/or functional ondemand extensions. Towards the end of its lifecycle, the selfgrowing network may still remain active and may serve as a dedicated purpose (embedded) network or as a failover for applications associated with other networks sharing the same area.

The Self-growing concept incorporates both collaborative and autonomic aspects. Cooperative behaviour and problem solving is critical in the initial phase of self-growing, that is the small-scale network, as well as in the evolution to a larger scale network, able to serve different purposes and larger systems.

In contrast to existing approaches for autonomous and selfconfiguring / self-managing networks, a self-growing network follows some rules of evolution along its lifecycle, following, a predetermined progression from purposes requiring a lower level of complexity towards purposes requiring a higher level of complexity regarding reconfiguration and cooperation capabilities. Thus, a self-growing network cannot freely evolve but is restricted towards an intended purpose. Nevertheless, the degree of freedom to deviate from a planned lifecycle is a matter of the purpose of a self-growing network. Morever, a Self-growing network will incorporate selflearning capabilities: self-growing policies and rules may be derived, for example, in case that a self-growing network has to address emergencies in the vicinity which can be translated to "temporary" purposes. In this scope, the optimal balance between the autonomic and cooperative paradigms may be different according to the purpose of the self-growing network. This will be reflected in the rules that govern the evolution of the network, favouring (and motivating) varying degrees of cooperation between the network elements. The following key elements define the self-growing attribute:

A lifecycle is defined as either self-determined or pre-planned path along a sequence of progression points that define (potentially temporary) stable points in the evolution of a selfgrowing network. Progression points can be associated with stable configurations of a network potentially providing different functionalities for a certain purpose of the network.

A progression point shall associate with a set of attributes which can be described each by a non-empty set of parameters. If a set of metrics is made available for these parameters, the progression point is measurable. An associated descriptive set of values of parameters then makes a progression point well defined. Since the transition from one progression point to the next along the lifecycle is measurable in terms of parameter changes, it also implicitly describes the benefit (or cost) obtained from an evolutionary step. [10] provides a more complete view on the self-growing key elements as well as exemplary transition and lifecycles.

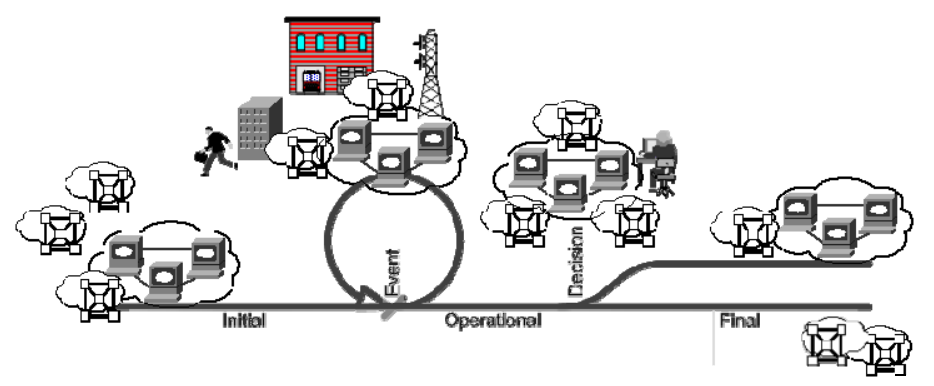

Figure 1: Self-Growing Network Life Cycle.

\section{Key SELF-Growing StUdy DiRECTIONS}

In the framework of CONSERN the folowing research axes are currently considered: (i) Scenarios, Use Cases, (ii) Requirements and Self-growing architecture, and (iii) Enablers and mechanisms for cooperative control. The folowing sections provide an overview of corresponding elaboration and progress.

\section{A. CONSERN Scenarios and Use Cases}

In the context of CONSERN scenarios describe application examples highlighting key benefits of CONSERN by attributing those to the scenario in an aggregated way. Five (5) high level scenarios have been identified [11] and elaborated in relation to the different scales and areas of applicability of CONSERN's key research directions (i.e. energy awareness and self-growing systems), namely, Construction Sites, Embedded Incident Area Network, Home and Office Environment, Urban Heterogeneous Environments, and Campus environment.

Construction Sites scenario addresses wide-area and infacility construction sites as well as moving work zones. Network evolution is achieved by cooperating, collaborating and integrating with neighboring networks and by continuously updating operational policies. Moving work zones are considered a special application of this concept in that they usually rely on short-living configurations with 
respect to a given geographical area. Road, railway or inland waterway construction scenarios may apply, explicitly requiring the use of mobile nodes and networks.

Embedded Incident Area Networks scenario addresses the deployment of a network in a limited geographical area and the network growing with upcoming requirements from the deployed area. In case of an exceptional event on demand reconfiguration capabilities allow to switch to a special purpose (focusing on safety). The temporary switch of purpose then will designate (part of) the network to implement an incident area network until the incident is resolved. This scenario thus illustrates self-adaptation on returning to preplanned operation and evolution.

Home and Office Environment scenario addresses the deployment of a heterogeneous wireless network in a limited geographical area, such as a home and office environment. in order to guarantee the provision of voice and data communication services, but also acts as a large scale, distributed and cooperating system for monitoring and control, possibly incorporating Wireless Sensor Networks. A particular challenge lies in the introduction of novel radio components needed to be included into the existing network by appropriate self-reconfiguration and self-adaptation of the currently deployed network environment by appropriate self-growing algorithms in an automated way as the owner of such an environment is assumed to be a person without any technical skills. A further challenge lies in the efficient inter-network parameterization, based on methods and algorithms to support spontaneous ad-hoc cooperation between objects and exploiting network-centric computing paradigms with dynamic resource discovery and management.

Urban Heterogeneous Environment scenario addresses the network deployment within an urban area with offices where people are working and homes where people are living. As people, on a daily basis, move between offices and homes the need for network resources grows and shrinks. The main objective of the scenario is to find ways to support the needed communication in an energy efficient manner. Cooperation between network nodes paves the way to adapt the utilization of resources to the traffic needs of the area at any one time.

Campus Environment scenario addresses a kind of evolution of a collection of independent home and office environments into a larger scenario, both in terms of number of nodes, area covered, coexistence problems encountered, or increasing collaboration and functional enhancements opportunities. The campus environment is characterized by a high density of wireless access nodes forming an individual micro- or femtocellular network. Even though deployment and operation of those networks is independent, it can assumed, in contrast to a home environment, the presence of a common single coordinating entity known to all deployed devices.

In the scope of the above presented elaborated scenarios a set of sixteen (16) technical use cases have been defined by the CONSERN partners adressing specific aspects related to energy aware, self-growing networking and thus linked to the project objectives. Such use cases highlight the whole diversity of the CONSERN related attributes including self- growing, energy awareness, situation awareness, cooperativeness, reconfigurability, autonomicity, dependability, spectral efficiency, system performance, adaptation efficiency through a set of specific parameters which are observable within each of the use cases.

\section{B. REQUIREMENTS AND SELF-GROWING ARCHITECTURE}

The requirements which have been identified within each technical Use Case's context have been further classified as functional and non-functional requirements. Functional requirements capture the intended behavior of the system which can be expressed as functions the system is required to perform, whereas non-functional requirements specify overall system characteristics and constraints which system need to satisfy. The folowing table summarises (high level) functional and non functional requirements.

\begin{tabular}{|l|}
\hline Functional Requirements \\
\hline Monitoring and measurements \\
\hline Information and knowledge dissemination and sharing \\
\hline Learning \\
\hline Network node detection and integration \\
\hline Decision Making \\
\hline Optimisation action application \\
\hline Non-fuctional requirements \\
\hline Constraints for low power \\
\hline Seamless operation \\
\hline Dynamically adjustable operation \\
\hline Dependable and efficient operation \\
\hline
\end{tabular}

Figure 2: CONSERN functional and non-fucntional requirements.

An initial set of functional blocks have been identified on the way towards formal specifications for interfaces, primitives, functional requirements, functional entities and their relationship and other elements needed to specify a concise framework:

- Sensor coordinator,

- Network configuration,

- Information exchange,

- Knowledge sharing,

- Information awareness/learning,

- Decision making,

- Central gateway device.

Main objective of this working axis is a dynamic cognitive control architecture suitable to "survive" major changes in underlying network topologies and capable to extend and learn from these changes as well as the specification of interfaces between cognitive functions that allow to dynamically add or remove functionality and to integrate these into an enclosing architecture.

\section{ENABLERS FOR COOPERATIVE OPERATION}

As already stated, a self-growing network needs to follow some rules of evolution along its lifecycle; additionally, a selfgrowing network evolves towards an intended purpose. In this sense, additional and totally new network management directions need to be taken into account in order to reflect: 
- The network lifecycle and the evolution along progression points,

- The purposes that can be served during a selfgrowing lifecycle,

- The corresponding rules for the network evolution, together with the respective actor which is liable for such rules, and,

- The environment of the network, in terms of neighbouring networks and network nodes that can be integrated in order to enable another purpose, or the embedded applications and services that can be added as a result of the new purpose that can be served.

A self-growing network or, more generally, a collection of collaborating networks can be tuned up towards a new purpose utilizing a suitable set of self-x capabilities for appropriate decision making and cross-network reconfiguration. A collection of collaborating networks thus becomes selfgrowing if it cooperatively applies its self-x capabilities for implementing a given (common) purpose. In this sense, cooperative decision and control under energy constraints is considered as key enabler towards energy-aware self-growing networks.

Implementing cooperation requires the deployment of communication enabling functionality. In this framework, cooperation enablers refer to those mechanisms, procedures and/or protocols which make the collaboration between network nodes and system entities possible. Key aspects for decision and control that are based on cooperation or can potentially benefit from it, include:

- Information exchange, ranging from node independent sensing (i.e., no information exchange between nodes) to full context/knowledge sharing,

- Coordination of decision and configuration control ranging from independent decisions up to fully coordinated decisions about reconfiguration actions,

- Utilisation of layer mechanisms for cooperative transmissions.

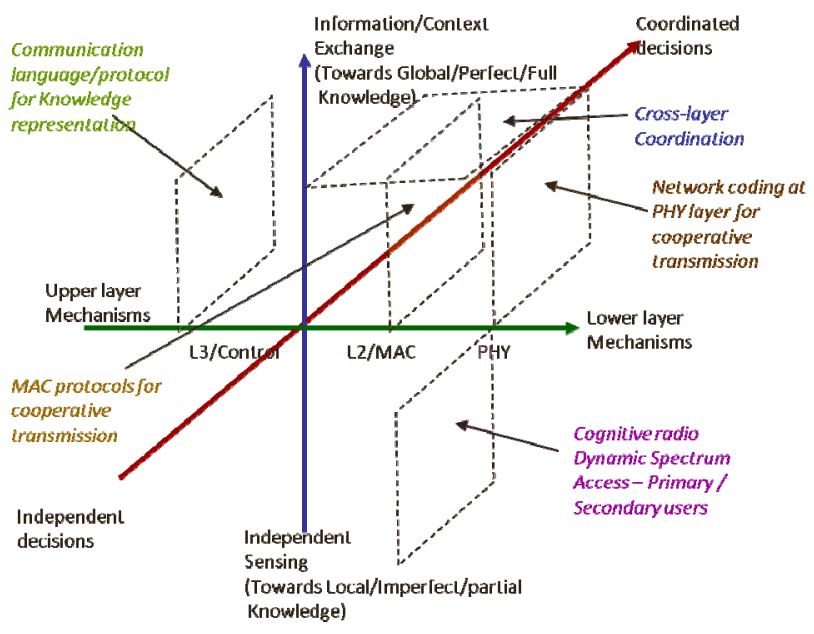

Figure 3: CONSERN 3-Dimensional cooperative decision and control approach.
In Fig.3, the above three different dimensions of cooperative operation are illustrated along with some key enabling technologies and solutions that can be deployed for addressing energy-efficiency. These technology solutions are depicted as planes in the three-dimensional space described above. The planes illustrated here are only examples that demonstrate the scope of cooperation.

In principle, any cooperative and/or autonomous solution can be mapped to this space which can present numerous kinds of solution arrangements for cooperation. In this sense, thedifferent ways of collaboration ranges from information exchanging information coupled with independent decision making to the deciding on a coordinated plan of configurations divided between different network nodes. Additional information on the CONSERN cooperation framework is available in [11]. Such 3-dimensional approach includes:

- Information/Context exchanging axis (or collaboration axis): sensing data, configuration settings, fused/processed information, knowledge presentation, etc.,

- Decision coordination and control axis (or coordination axis): routing/relaying control, negotiation protocol, coordination planning, synchronisation, distributed decision making, knowledge reasoning, conflict resolution, etc.,

- Layer mechanisms axis (or communication layer axis): Routing/ relaying at L3 layer, MAC protocols and/or relaying at L2 layer, cooperative multi-point transmission at L1 (PHY) layer, network coding and cross-layer etc.

CONSERN elaborates on corresponding cooperation enablers and mechanims featuring:

- Mechanisms for cooperative adaptation of elements to the most energy efficient pattern utilizing knowledge of neighbouring elements,

- Adaptive distributed solutions for decision making for reducing power consumption based on selforganising networks and mobile relay stations,

- Mechanisms for discovering co-located networks and negotiating network parameters between co-located networks in order to enable cross-network communication sharing of network resources,

- Low power physical layer network coding algorithms and techniques to efficiently communicate between different network objects, while taking into account their effect on other communication links in the vicinity,

- Advanced neighbouring relation mechanisms for discovering and setting up communication links to nodes handling a neighbouring coverage area to in order to support the cognitive control functionality.

\section{OVERVIEW ON CONSERN WORKING DIRECTION}

In order to efficiently address the upper presented research directions, the project is organized based on the following structure: 
WP1 addresses high level and system topics related to the provision of improved energy efficiency networking solutions and building on novel self-growing network concepts, as well as a holistic impact assessment and more applied market evaluation on the expected gains. Initially, scenarios will be identified, specific use cases will be proposed and requirements will be defined. The expected gains from novel energy efficiency and self-growing network solutions will be quantified using existing and new system level metrics which are elaborated in cooperation with WP2-WP4 with the objective to study their impact from a wider socio-economic impact perspective and identify the potential gains for business players. Finally, Dissemination and Exploitation and Standardisation actions are also coordinated by WP1.

WP2 focuses on aspects related to energy efficiency and optimisation, covering system or network wide optimisations, terminal or architectural optimisations, then joint optimisations and further improvements using run-time selfadaptation techniques. All node-level techniques for energy optimisation that are not explicitly cooperative fall in this WP2.

WP3 targets cooperation and collaboration enablers and mechanisms, cooperative decision and control algorithms, as well as all techniques that maximize the expected gain explicitly by utilizing cooperative behaviour. The relation between autonomic capabilities and cooperative decision and control will also be investigated in WP3 in order to maximize the expected gains for energy consumption and dependability. WP4 deals with self-growing network paradigms, defining and developing the enablers that will pave the way for such paradigms. Initially, potential benefits and challenges will be formalized, the generic requirements of WP1 will be refined for explicit scalable self-growing networks of heterogeneous nodes and deployment stages will be outlined. Based on this exercise, missing functionalities will be identified and steps towards developing the corresponding "bricks" will be initiated, building on autonomic and collaborative mechanisms studied in other WPs.

WP5 will focus on the validation and the proof-of-concept of the solutions proposed in all other WPs. A selected but representative and significant subset of the proposed techniques will be evaluated on a test-bed environment in order to present tangible outcomes that showcase the validity of CONSERN solutions.

\section{CONCLUSIONS}

This paper details novel Self-Growing related research directions as they are considered within the FP7 Research Project CONSERN. The corresponding results are expected to enable an efficient, future framework in which mobile networks adapt their purpose to evolving needs over the whole life-time. As a consequence, network equipment will continuously and autonomously adapt to a changing and evolving environment. As a result, the overall system efficiency will be autonomously optimized in terms of energy efficiency, spectral resource usage efficiency, etc.

\section{ACKNOWLEDGEMENT}

The research leading to these results was derived from the European Community's Seventh Framework Programme (FP7) under Grant Agreement number 257542 (CONSERN).

\section{REFERENCES}

[1] Nokia Siemens Networks Unite, Energy efficiency 2.0, available at: http://unite.nokiasiemensnetworks.com/adwords/article/view/id/4.

[2] Y. Agarwal, C. Schurgers and R. Gupta, "Dynamic Power Management Using on Demand Paging for Networked Embedded Systems", Proceedings of the 2005 conference on Asia South Pacific Design Automation, Shanghai, China, Page(s): 755 - 759, 2005.

[3] M. Sgroi, et al. "Synthesis of Embedded Software Using Free-Choice Petri Nets", in Proceedings of Design Automation Conference, DAC '99. 1999. New Orleans, USA.

[4] M. Sgroi, L. Lavagno, and A. Sangiovanni-Vincentelli, "Formal Models for Embedded System Design", in IEEE Design \& Test Magazine. Special Issue on System Design. 2000.

[5] G. R. Callou, et al. "A coloured Petri net based approach for estimating execution time and energy consumption in embedded systems", in Proceedings of the 21st Annual Symposium on integrated Circuits and System Design SBCCI '08. 2008 Gramado, Brazil: ACM, New York, NY.

[6] M. N. O Júnior, et al. "Towards a Software Power Cost Analysis Framework Using Colored Petri Net", in Integrated Circuit and System Design: Power and Timing Modeling, Optimization and Simulation, 14th International Workshop, PATMOS 2004, 2004, Santorini, Greece: Springer Berlin / Heidelberg.

[7] M. N. O. Junior et al. "Analyzing Software Performance and Energy Consumption of Embedded Systems by Probabilistic Modeling: An Approach Based on Coloured Petri Nets" in 27th International Conference on Applications and Theory of Petri Nets and Other Models of Concurrency ICATPN 2006. 2006 Turku, Finland.

[8] A. Leonardo et al., "Mapping live sequence chart to coloured petri nets for analysis and verification of embedded systems", SIGSOFT Softw. Eng. Notes, 2006. 31(3): p. 1-25.

[9] H. Kevin,F. Christian, and H. Reinhold, "Towards formally verifiable resource bounds for real-time embedded systems", SIGBED Rev., 2006. 3(4): p. 27-36.

[10] B. Bochow and M. Emmelmann, "Purpose-driven, Self-growing Networks - a framework for enabling cognition in systems of systems", in VTC Spring 2011 W2: Second Green Wireless Communications and Networks Workshop (GreeNet), to appear

[11] G. Koudouridis, G. Hedby, W. H. Chin, A. Merentitis, M. Stamatelatos, N. Alonistioti, O. Yaron, "On the Enablers for Energy-Aware Networking and Cooperative Decision Control", in VTC Spring 2011, W3: Cognitive radio and Cooperative strategies for POWER saving (C2POWER), to appear. 